\title{
Travailler en coopérative et dans l'économie sociale, effets sur la satisfaction et le sens du travail
}

Working in a cooperative and social economics: Effects on job satisfaction and the meaning of work

Trabajar en cooperativa y en la economía social, efectos sobre la satisfacción y el sentido del trabajo

Davy Castel, Claude Lemoine et Annick Durand-Delvigne

\section{CpenEdition}

Édition électronique

URL : http://journals.openedition.org/pistes/1788

DOI : 10.4000/pistes. 1788

ISSN : 1481-9384

Éditeur

Les Amis de PISTES

Édition imprimée

Date de publication : 1 novembre 2011

Référence électronique

Davy Castel, Claude Lemoine et Annick Durand-Delvigne, «Travailler en coopérative et dans l'économie sociale, effets sur la satisfaction et le sens du travail », Perspectives interdisciplinaires sur le travail et la santé [En ligne], 13-2 | 2011, mis en ligne le 01 novembre 2011, consulté le 30 avril 2019. URL : http://journals.openedition.org/pistes/1788; DOI : 10.4000/pistes.1788

Ce document a été généré automatiquement le 30 avril 2019.

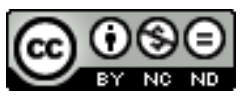

Pistes est mis à disposition selon les termes de la licence Creative Commons Attribution - Pas d'Utilisation Commerciale - Pas de Modification 4.0 International. 


\title{
Travailler en coopérative et dans l'économie sociale, effets sur la satisfaction et le sens du travail
}

\author{
Working in a cooperative and social economics: Effects on job satisfaction and \\ the meaning of work \\ Trabajar en cooperativa y en la economía social, efectos sobre la satisfacción y el \\ sentido del trabajo
}

Davy Castel, Claude Lemoine et Annick Durand-Delvigne

\section{Introduction}

1 Selon les acteurs concernés (réseau SCOP entreprises, représentant les sociétés coopératives et participatives de France) et la presse spécialisée (Participer; Alternatives économiques, 2006), le management coopératif entraînerait une augmentation importante de la qualité de vie au travail et de la satisfaction ressentie par les individus. Peu après la naissance en France du Conseil Supérieur de l'Économie Sociale chargé de promouvoir ce secteur de l'économie (décret du 11 juillet 2006), il est sans doute pertinent de s'interroger sur les effets réels de l'économie sociale sur la qualité de vie et plus spécifiquement sur la satisfaction des individus au travail. En effet, la qualité de vie au travail au sein de l'économie sociale, sa capacité à répondre aux attentes des individus et à être source de satisfaction n'ont fait l'objet que de très peu d'études à ce jour. Quelles sont les conséquences du fait de travailler dans l'économie sociale sur la satisfaction exprimée par les salariés? Quelle est la place de l'économie sociale dans les satisfactions et insatisfactions exprimées? L'organisation démocratique des entreprises qui caractérise l'économie sociale n'a-t-elle pas aussi des effets négatifs? Dans cette étude réalisée en France, nous avons procédé à l'analyse qualitative des liens entre spécificités de l'économie sociale et satisfaction au travail. Les conclusions contribueront à déterminer si 
l'économie sociale représente un modèle d'organisation du travail source de satisfaction au travail, et si oui, à identifier les facteurs explicatifs pour en favoriser le développement dans le monde du travail en général.

\subsection{La satisfaction au travail}

2 La satisfaction au travail correspond à l'attitude de l'individu à l'égard de son activité et de son environnement professionnel (Weiss, 2002) ; elle dépend du niveau de congruence entre les caractéristiques du travail perçues et les attentes de l'individu (Bretz et Judge, 1994 ; Dawis, 1992 ; Kristof-Brown, Zimmerman et Johnson, 2005). Depuis la théorie bifactorielle d'Herzberg $(1966,1978)$, on distingue deux familles de déterminants de la satisfaction et de l'insatisfaction au travail : les facteurs intrinsèques, qui correspondent aux caractéristiques de l'activité de travail elle-même, et les facteurs extrinsèques, qui correspondent aux caractéristiques de l'environnement dans lequel s'effectue l'activité. Les facteurs intrinsèques sont les suivants : accomplissement par le travail, considération reçue, intérêt pour la tâche, responsabilités, perspectives d'évolution, niveau d'informations sur la tâche, autonomie, utilité du travail, diversité des tâches, sentiment d'autoefficacité et utilisation des compétences personnelles. Les facteurs extrinsèques sont la politique d'entreprise, les compétences de l'encadrement, le salaire, les conditions de travail, le temps de travail, la stabilité de l'emploi, la sécurité au travail, la charge de travail et la pression ressentie, les relations interpersonnelles, le statut et le prestige de l'emploi (Herzberg, 1978; Johnson et Johnson, 2002 ; Ritter et Anker, 2002 ; Souza-Pouza et Souza-Pouza, 2000; Spector, 1997). La satisfaction au travail a des implications importantes à différents niveaux : elle peut diminuer les intentions de quitter l'emploi (Tett et Meyer, 1993), l'absentéisme et l'épuisement émotionnel (Vandenberghe, Stordeur et D'Hoore, 2009), augmenter la productivité (Iaffaldano et Muchinsky, 1985) et l'engagement organisationnel (Van Knippenberg et Van Schie, 2000). Elle est un indice significatif du niveau global de bonheur (Argyle, 2001). Dans une étude de l'institut de sondage Ipsos commandée par le Centre d'analyse stratégique en novembre 2009 (organisme rattaché au premier ministre ayant pour mission d'éclairer le gouvernement français dans la définition de ses orientations stratégiques), $99 \%$ des personnes interrogées signalent qu' « apprécier son travail au quotidien » est un facteur important pour leur santé mentale; il s'agit du déterminant le plus fréquemment cité. Un niveau élevé de satisfaction au travail est une condition nécessaire à l'obtention d'un état de santé optimal, caractérisé par l'équilibre entre les demandes et les contributions respectives de la personne et de son environnement (définition du ministère de la Santé canadien, 1988). L'amélioration de la satisfaction dans le travail est donc un enjeu majeur, d'autant plus d'actualité que la demande d'investissement du salarié de la part de l'entreprise apparaît de plus en plus importante. Le déni du mécontentement et l'absence de solutions dans un tel contexte conduirait à la fois à des dysfonctionnements importants pour l'entreprise, mais aussi à des souffrances pour les salariés. Les pouvoirs publics semblent peu à peu en prendre conscience puisque, à titre d'exemple, la satisfaction des travailleurs vis-à-vis de l'emploi est le premier d'une série de dix objectifs formulés par les dirigeants européens lors des sommets de Lisbonne (2000) et Laeken (2001) pour améliorer la qualité de vie au travail. 


\subsection{L'économie sociale}

3 Les institutions relevant de l'économie sociale développent des activités économiques régies par les quatre principes suivants (Defourny, 1992) : finalité de service aux membres ou à la collectivité plutôt que de profit, autonomie de gestion, processus de décision démocratique et primauté des personnes et du travail sur le capital dans la répartition des revenus. Au 31 décembre 2006, l'économie sociale représente $10 \%$ du PIB, $9 \%$ des entreprises et $10 \%$ des emplois (soit 2,1 millions de travailleurs) en France (Conseil National des Chambres Régionales de l'Économie Sociale, 2009). Elle réunit les associations, les mutuelles et les coopératives. Des trois composantes, les associations forment la branche la plus répandue et représentent à elles seules $85 \%$ des établissements et $80 \%$ des emplois de l'économie sociale (soit 1,7 millions de travailleurs). Elles se distinguent des deux autres composantes par le fait que les membres bénévoles y sont généralement plus nombreux que les membres salariés; la dimension économique de l'activité réalisée y est souvent moins importante qu'elle ne l'est dans les mutuelles et la majorité des coopératives. Par ailleurs, dans les associations, les bénéfices éventuels ne peuvent être redistribués aux membres (à la différence des mutuelles et des coopératives de production qui, si leur finalité n'est pas le profit, ne sont pas pour autant soumises à cette contrainte de non-distribution). Les mutuelles, organismes chargés de la gestion collective des risques auxquels leurs assurés sont exposés, se distinguent des sociétés d'assurance privées par le fait qu'elles sont contrôlées par leurs sociétaires (les assurés eux-mêmes ou leurs représentants). Elles emploient 118000 personnes, soit $6 \%$ des emplois de l'économie sociale (CNCRES, op. cit.). Les coopératives sont principalement de trois types : les coopératives de production (SCOP) où l'entreprise appartient aux salariés coopérateurs, les coopératives d'usagers ou de consommateurs, et les coopératives d'entreprises qui regroupent des exploitants, des commerçants ou des artisans qui mutualisent des moyens pour être plus solides ensemble.

Toutes les composantes de l'économie sociale ne sont pas stricto sensu des organisations de travail. La majorité des associations et les coopératives de consommateurs entrent difficilement dans cette catégorie, soit qu'on les classe - à tort - dans l'économie sociale alors qu'elles ne développent pas d'activité économique, soit que l'activité qu'y mènent les individus ne constitue pas leur activité de travail principale. Ces organisations apparaissent donc peu pertinentes comme champ d'investigation de la satisfaction au travail dans l'économie sociale. Dans les coopératives d'entreprises, les entreprises coopèrent entre elles sans que le statut coopératif n'ait nécessairement de conséquences sur les modes d'organisation au sein de chacune des entreprises membres. Restent alors les mutuelles, les banques coopératives et les coopératives de production comme possibles lieux d'étude de l'impact de l'économie sociale sur la satisfaction. Cependant, parce que leurs sociétaires sont avant tout leurs clients ou assurés et non leurs salariés, les banques coopératives et les mutuelles peuvent appliquer les principes d'économie sociale sans que cela ne se ressente fortement chez leurs salariés. Les sociétés coopératives de production (SCOP) sont donc les structures d'économie sociale où le management participatif est susceptible d'avoir le plus d'influence sur la vie au travail des salariés. Nous avons donc choisi les SCOP comme composante la plus adéquate pour étudier l'impact de l'économie sociale sur la satisfaction au travail. Cela signifie que si les particularités de l'économie sociale ont un impact, positif ou négatif, sur la satisfaction au travail, c'est dans les SCOP que ces effets seront les plus à même d'être observés. Cela 
signifie aussi que la prudence s'impose lors de la généralisation des résultats obtenus à partir des SCOP aux autres composantes de l'économie sociale.

\subsection{Les sociétés coopératives ouvrières de production (SCOP)}

5 Les SCOP développent un style de management coopératif visant à réduire la frontière entre conception et exécution en s'articulant autour du principe de travailleur-associé : les travailleurs ne sont pas uniquement des salariés de l'organisation, mais en sont propriétaires et participent à l'ensemble des décisions. L'Alliance Coopérative Internationale (ACI) donne cette définition de la coopérative : « une association autonome de personnes volontairement réunies pour satisfaire leurs aspirations et besoins économiques, sociaux et culturels communs au moyen d'une entreprise dont la propriété est collective et où le pouvoir est exercé démocratiquement. » En tant qu'entreprises d'économie sociale, les SCOP n'ont pas pour finalité la seule rentabilité économique, mais aussi l'utilité et la responsabilité sociale ainsi que le développement du milieu socioéconomique dans lequel elles s'insèrent. La priorité est donnée au travail et à l'emploi dans la répartition des richesses créées, plutôt qu'au capital et à la distribution de bénéfices. Dans leurs relations avec le milieu économique, les SCOP cherchent à préserver leur indépendance et le libre-choix de leurs membres et à ne pas s'assujettir aux seules règles du marché (même si, légalement, elles sont soumises aux mêmes règles économiques que toutes les autres entreprises). L'entreprise est la propriété des salariés qui doivent donc participer individuellement à son capital en devenant associés (tous ne le sont pas nécessairement, même s'ils ont tous vocation à l'être; dans certaines SCOP c'est obligatoire au bout de deux ans). Au sein de l'entreprise, le pouvoir est exercé démocratiquement entre les salariés-associés selon le principe " un Homme = une voix ». Fondée sur ce droit de vote, l'égalité entre les salariés vient suppléer l'inégalité fondée sur les statuts hiérarchiques. Le ou les gérants de la SCOP sont élus par et parmi les salariés-associés. Les règles internes sont fondées sur une délibération collective et une adhésion volontaire davantage que sur la soumission à une autorité. Enfin, les informations ont vocation à être partagées entre tous les membres, et non la propriété de la direction.

6 En France, la SCOP est une entreprise de forme société anonyme (SA) ou société anonyme à responsabilité limitée (SARL) dont les salariés sont associés majoritaires. En 2009 on dénombre 1925 SCOP en France (contre 1600 en 2005) soit 3,9 milliard d'euros de chiffre d'affaires et 40424 emplois (contre 35000 en 2005 et 28000 en 1998). La taille moyenne d'une SCOP est de 21 salariés, toutefois cette moyenne masque des disparités importantes, $60 \%$ des SCOP étant des micro-entreprises inférieures à 9 salariés. Les secteurs d'activité les plus représentés par les SCOP sont les services ( $46 \%)$, l'industrie $(23,5 \%)$ et le bâtiment ( $29 \%$ ). Le secteur du bâtiment et le secteur des services intellectuels (bureaux d'étude, conseil et ingénierie) sont les plus créateurs d'emplois nouveaux. En moyenne $67,5 \%$ des salariés sont associés et $80 \%$ le deviennent après deux ans d'ancienneté. Les femmes occupent la majorité des emplois en SCOP $(60 \%)$ comme dans les autres composantes de l'économie sociale, ce qui contraste avec le secteur privé hors économie sociale où la proportion est inversée. Cependant, comme dans le secteur privé et à la différence des autres composantes de l'économie sociale, les hommes sont largement majoritaires parmi les cadres et dirigeants des coopératives ( $75 \%$ ). La répartition par catégorie socioprofessionnelle (CSP) montre une structure d'emploi en coopérative assez 
singulière avec peu d'employés et une très forte proportion de professions intermédiaires (près de $40 \%$ ). La proportion des salariés de plus de 50 ans $(30 \%)$ est légèrement supérieure en coopérative par rapport au secteur privé hors économie sociale, et comparable à celle du secteur public. Enfin, notons qu'une grande proportion des emplois en coopérative sont des emplois à temps plein (plus de $80 \%$ ), ce qui les distingue fortement des autres composantes de l'économie sociale, notamment des associations (CNCRES, 2009 ; réseau SCOP entreprises, 2010).

\subsection{Effets attendus sur la satisfaction au travail}

7 Sur la majorité des facteurs intrinsèques, on s'attend à ce que la satisfaction au travail soit élevée en SCOP et que les principes d'économie sociale soient au cœur des satisfactions exprimées. La réduction de la séparation entre conception et exécution, l'augmentation de la maitrise du processus de travail, la plus grande mise à contribution des compétences de chacun, la recherche d'un travail utile à la société, l'égalité de chacun face aux décisions et à l'information, et le fait de travailler pour soi devraient augmenter la satisfaction provenant du travail lui-même. Sur de nombreux aspects extrinsèques, la satisfaction devrait également être importante en SCOP : culture d'entreprise davantage partagée, renforcement de la confiance en l'encadrement à cause de son élection, plus grande sensibilité à l'amélioration des conditions de travail et d'emploi du fait de la priorisation de la responsabilité sociale de l'entreprise et de la prise de décision collégiale. Cependant, la relation attendue entre économie sociale et satisfaction apparaît plus mitigée sur de nombreux autres aspects. Ainsi, la pression ressentie peut être plus faible et moins source d'insatisfaction grâce à un plus grand nombre de ressources pour y faire face (autonomie, marges de manœuvre), mais l'augmentation des responsabilités des salariés-associés peut également accentuer les exigences de l'activité. De même, les relations interpersonnelles peuvent s'améliorer du fait d'une plus grande égalité, mais peuvent également se détériorer à cause de l'ambiguïté des rôles (salariés et dirigeants à la fois) et de la plus grande probabilité d'apparition de conflits résultant de l'augmentation des pouvoirs (et des contre-pouvoirs) de chacun.

\section{Méthode}

\subsection{Participants et lieux de recueil des données}

8 Nous avons décidé d'étudier la satisfaction au travail au sein de deux entreprises, l'une SCOP, l'autre entreprise SARL classique, qui ne différaient autant que possible que selon cette dimension. Elles ont donc été choisies dans le même secteur d'activité, celui du conseil et de la communication en développement durable. Parmi les différents secteurs d'activité dans lesquels les SCOP sont bien représentées en France, celui des services intellectuels est le plus important ( $33 \%$ des SCOP en France; réseau SCOP entreprises, 2010). Au sein de ce dernier, le sous-secteur du conseil et de la communication est le plus créateur d'emplois nouveaux, avec une croissance moyenne annuelle de $+8,5 \%$ entre 2001 et 2006. De plus, ce secteur est particulièrement bien représenté parmi les SCOP du Nord de la France où s'est déroulée cette étude. Cependant, c'est aussi dans ce secteur d'activité que les SCOP de petite taille sont les plus nombreuses, avec $82 \%$ de micro-entreprises inférieures à 9 salariés et une taille moyenne des SCOP dans ce secteur de 15 salariés 
contre 21 tous secteurs d'activité confondus (CNCRES, 2009; réseau SCOP entreprises, 2010).

Afin de sélectionner deux entreprises dans ce secteur, nous avons premièrement recherché une SCOP en vue de participer à l'étude, car elles sont évidemment en nombre inférieur aux entreprises privées, puis nous avons cherché une entreprise privée qui lui était aussi similaire que possible sur les critères autres que le type de structure, comme la taille de l'entreprise ou son activité principale. Via le réseau SCOP entreprises et l'APES Nord-Pas De Calais (Assemblée Permanente de l'Économie Sociale) nous avons obtenu la liste des SCOP de ce secteur d'activité puis opéré une première sélection en fonction de deux critères, la taille de l'entreprise (nous recherchions une SCOP de cinq salariés au moins, beaucoup n'atteignant pas ce critère) et la localisation, accessible pour les enquêteurs. Nous avons ensuite contacté les entreprises retenues par courriel afin de leur proposer de participer à l'étude. L'une d'elles s'est montrée intéressée dans des délais plus courts que les autres. Les entreprises privées exerçant dans le même domaine précis d'activité et de même taille ont ensuite été passées en revue. Une entreprise privée très similaire à la SCOP, y compris par son lieu d'implantation géographique et son rayon d'action, a été retenue.

10 La SCOP retenue est une coopérative composée de sept salariés au moment de l'étude (une assistante de direction, cinq consultant(e)s dont l'une est également gérante et une chargée de mission), créée en 1993 et spécialisée dans la conduite de projets de développement durable. Les participants membres de la coopérative sont donc sept, cinq femmes et deux hommes, âgés de 24 à 47 ans (moyenne: 32 ans). Les niveaux de qualification varient du BEP (diplôme professionnel) au doctorat et l'ancienneté dans l'entreprise varie de 3 mois à 4 ans (m.: 2 ans). Aucun des salariés n'avait travaillé en SCOP auparavant et peu avaient de l'expérience dans le domaine du conseil et de la communication avant d'intégrer cette SCOP. Cinq salariés sur sept sont associés de la coopérative ; deux pourront le devenir lorsqu'ils auront atteint les six mois d'ancienneté requis pour devenir sociétaire. Enfin, six salariés sont à temps plein et un seul à mitemps.

11 L'entreprise privée retenue est une agence de conseil en communication publique créée en 1990, spécialisée dans les projets liés à la décentralisation, au renouvellement urbain et au développement durable. Cette entreprise est composée de six membres dont la créatrice et directrice de l'agence, un directeur de création, deux consultants, une chef de projet et une assistante. L'un des deux consultants n'ayant pu participer à la recherche pour cause de congés, les participants membres de l'entreprise privée sont donc cinq, trois femmes et deux hommes, âgés de 27 à 45 ans (m.: 38 ans). Les niveaux de qualification varient du baccalauréat (diplôme de fin d'études secondaires) au master et l'ancienneté dans l'entreprise varie de 7 mois à 17 ans (m.: 7,25 ans). Tous ont de l'expérience dans le domaine dans lequel ils exercent (12,4 ans en moyenne). La directrice de l'agence et le directeur de création sont actionnaires de l'entreprise. Enfin, tous les salariés sont à temps plein.

12 Les deux entreprises ne diffèrent ni par la taille ni par l'activité, deux caractéristiques influentes sur la satisfaction. Une première différence apparaît dans les métiers représentés, puisque la SCOP est composée quasi exclusivement de consultants tandis que les métiers représentés dans l'entreprise privée sont plus divers. Cependant, cette différence est une conséquence directe du type de structure et du choix de la SCOP de ne pas établir de hiérarchie aussi saillante que dans les entreprises privées. L'autre 
différence se situe au niveau des facteurs ancienneté et expérience professionnelle. Leurs différences d'ancienneté et d'expérience peuvent amener les individus à exprimer des attentes différentes, et donc des niveaux de satisfaction différents, et peuvent peser sur le mode d'organisation du travail. Ces différences peuvent constituer des sources d'influence à prendre en compte au moment de l'interprétation des résultats. Les autres variables démographiques ne montrent pas de différences majeures entre les deux entreprises.

\subsection{Outils de mesure}

13 Nous avons fait le choix de procéder à une analyse qualitative par le biais d'entretiens semi-directifs. Cela nous a permis d'analyser finement les facteurs explicatifs des sources de satisfaction et d'insatisfaction exprimées, et d'établir le rôle joué par l'économie sociale et ses spécificités. Le guide des entretiens semi-directifs a été construit sur la base de deux questions destinées aux participants: " quels sont dans votre travail actuel vos principaux facteurs de satisfaction et pourquoi ? » et « quels sont dans votre travail actuel vos principaux facteurs d'insatisfaction et pourquoi? » Les entretiens duraient de 30 à 45 minutes, les participants n'étant pas interrompus, l'entretien s'arrêtant quand les participants estimaient qu'ils avaient entièrement répondu aux deux questions. Ainsi, chacun pouvait citer autant de facteurs de satisfaction et d'insatisfaction qu'il le jugeait pertinent. Les entretiens étaient enregistrés et soumis par la suite à une analyse de contenu thématique avec méthode des juges. Il s'agissait de repérer les facteurs de satisfaction et d'insatisfaction présents dans le discours des participants et le rôle de l'économie sociale dans ces facteurs. Le codage des retranscriptions d'entretiens en facteurs de satisfaction et d'insatisfaction a été effectué indépendamment par trois personnes différentes puis comparées afin de vérifier l'accord interjuges et la pertinence des catégorisations effectuées. La fréquence d'occurrence d'un facteur donné ainsi que son ordre d'apparition dans les sources de satisfaction et d'insatisfaction exprimées étaient ensuite utilisés comme indicateurs du poids de ce facteur dans le niveau général de satisfaction au travail.

Par ailleurs, il était également demandé aux participants d'évaluer leur niveau global de satisfaction/insatisfaction au travail sur une échelle graduée de 1 à 100.

\subsection{Procédure}

15 Les entretiens ont été proposés aux salariés sur la base du volontariat, cherchant à intéresser les participants dans une recherche sur leurs sources de satisfaction et d'insatisfaction au travail afin qu'ils s'en saisissent comme d'une opportunité de réflexion sur soi, plutôt que comme une contrainte ou un danger lié à la communication d'informations personnelles. En un mot, nous avons cherché, à travers le mode d'insertion du questionnement sur le terrain d'étude, à diminuer les sources de contreemprise et à provoquer une auto-emprise constructive (Lemoine, 2007). Dans ce but, il était dit en amont aux participants que l'entretien porterait sur leurs sources de satisfaction et d'insatisfaction; toutefois, il n'était aucunement fait mention, ni durant l'entretien ni avant, de l'existence d'hypothèses en lien avec l'économie sociale, afin de ne pas influencer les réponses des individus. 
16 Le recueil des données a été effectué à un mois d'intervalle dans les deux entreprises. Les entretiens semi-directifs ont eu lieu avec chacun des participants individuellement. Pour des raisons d'indisponibilité ils n'ont pu être menés qu'auprès de six des sept salariés de la coopérative. Ils ont été réalisés avec les six salariés de l'entreprise privée. Aucun évènement isolé n'est venu influencer les réponses des participants pendant ou juste avant les entretiens. Ils ont été menés au cours de la journée de travail, dans le calme et sans présence d'un tiers. L'enregistrement ne semble pas avoir été un frein à l'expression de la parole.

\section{Résultats}

\subsection{Analyse des entretiens}

\subsubsection{Satisfactions et insatisfactions au sein de la SCOP}

Le premier facteur de satisfaction, exprimé par tous les salariés de la SCOP (6/6), est la cohésion du collectif de travail. Par cohésion, les salariés entendent un haut degré de proximité perçue entre eux ainsi qu'un niveau élevé de convergence de buts et de valeurs. La cohésion du collectif de travail ne serait pas une conséquence de la gouvernance collégiale, mais de la proximité d'âge entre les salariés ainsi que de leur histoire commune dans l'entreprise. Selon eux, la relation est inverse : c'est parce que le groupe est cohésif que la collégialité fonctionne bien. Nous reviendrons sur la relation entre cohésion et collégialité lors de la discussion.

Le deuxième facteur de satisfaction exprimé par cinq salariés sur six (5/6) est la structure coopérative (SCOP) elle-même pour les raisons suivantes: un niveau d'autonomie et de responsabilité accru, et surtout un niveau équivalent de responsabilités pour chacun (coentrepreneuriat) ; la valorisation de la collaboration plutôt que de la compétition; la gouvernance collégiale, qui "rend réelle la co-élaboration", encourage à donner une place importante à chacun, et facilite l'intégration des nouveaux salariés; la considération obtenue par chacun des membres, présentée comme exceptionnelle par ceux qui avaient travaillé auparavant en entreprise privée; l'égalité des statuts; la politique salariale "humanisée » (les salariés ont le sentiment que leurs intérêts sont perçus et traités comme prioritaires).

19 La troisième source de satisfaction exprimée (3/6) est l'activité de travail elle-même (intérêt, utilité, diversité). Le domaine dans lequel cette activité s'inscrit (développement durable) semble avoir un impact important sur l'intérêt et l'utilité ressentie. L'activité de travail est jugée utile, car l'entreprise respecte ses responsabilités sociales autant qu'économiques et que l'activité menée est en conformité avec les principes d'économie sociale (sentiment de conformité entre actions et convictions).

20 Le premier facteur d'insatisfaction dans la SCOP, mis en avant par quatre salariés sur six (4/6), est la fonction d'animateur de réunions publiques, tâche importante dans l'activité de cette SCOP. Dans un cas, c'est la fonction elle-même qui pose problème. L'opérateur présente une attitude positive vis-à-vis de la fonction (utilité, pertinence) mais ne s'y sent pas à l'aise. Dans les autres cas, ce sont les déplacements tardifs et l'allongement des journées de travail que l'animation des réunions publiques (qui se tiennent en soirée) occasionne qui est source d'insatisfaction. Cela entraîne un envahissement de la sphère privée et familiale par le travail, d'autant plus saillant ici que la SCOP est composée 
majoritairement de femmes et que l'articulation vie professionnelle-vie familiale reste encore un problème principalement féminin (Durand-Delvigne, Verquerre et Masclet, 2000).

21 Le deuxième facteur d'insatisfaction (2/6) est la face cachée de la cohésion du collectif de travail citée en satisfaction. Cette cohésion apparait inégale et envahissante. Inégale, car les plus anciens forment inévitablement un noyau auquel tentent de se rattacher les opérateurs arrivés ensuite. Envahissante, car la forte cohésion amène le collectif à «raisonner en vase clos", professionnellement, puisque la SCOP travaille systématiquement avec des réseaux qui partagent les valeurs d'économie sociale, mais aussi hors travail puisque, selon les salariés, leurs comportements et leurs relations sont également déterminés par les valeurs auxquelles ils s'identifient.

Le troisième facteur d'insatisfaction tient à la répartition des tâches au sein de l'entreprise (2/6). Les consultants doivent consacrer un temps important à des tâches administratives considérées annexes malgré la création d'un poste d'assistant de direction, ce qui génère des conflits et des incompréhensions de part et d'autre. Si les consultants estiment que les tâches administratives devraient davantage reposer sur l'assistant de direction, ce dernier estime que les consultants n'ont pas conscience de l'ampleur de sa charge de travail.

23 Le dernier facteur d'insatisfaction (2/6) est la rémunération, car les salaires ne correspondent pas aux qualifications. Les opérateurs considèrent, en comparant avec des salariés d'autres entreprises, qu'ils ont dû faire un choix entre salaire et intérêt du travail. Ils ne regrettent cependant pas ce choix et par conséquent ne donnent pas un caractère prioritaire au salaire. Par ailleurs, le contrôle exercé par les coopérateurs sur leur niveau de salaire diminuerait selon eux l'insatisfaction ressentie, car ils font euxmêmes le choix (si c'en est un) de ne pas s'augmenter, la situation financière de la SCOP ne le permettant pas.

\subsubsection{Satisfactions et insatisfactions au sein de l'entreprise privée}

$\mathrm{Au}$ sein de l'entreprise privée, la culture d'entreprise est la source principale de satisfaction au travail, citée par l'ensemble des salariés (5/5). Elle repose sur trois valeurs fondamentales : « la liberté », « la complémentarité » et « la vérité ».

La liberté est celle que s'autorise l'entreprise dans le choix des projets sur des critères autres que les critères économiques. C'est ainsi que les salariés entrevoient la possibilité de donner un sens à leur travail. Cette liberté distingue l'entreprise, selon ses salariés, des autres entreprises privées de communication. C'est aussi l'application de la liberté à l'interne (choix des modes opératoires, maîtrise du temps de travail) qui est source de satisfaction.

La complémentarité renvoie à l'interdisciplinarité pratiquée dans l'agence : les opérateurs viennent de formations différentes et l'activité s'organise autour de leurs compétences complémentaires. Ce fonctionnement est source de satisfaction, car il est source d'enrichissement personnel et élève le niveau de traitement des projets.

La valeur vérité correspond à l'exigence de sincérité dans la communication, à la priorité donnée à la vérité sur le rentable. Elle consiste à faire travailler ensemble experts et communicants afin que la communication viennent vanter les vertus réelles du projet sans en cacher les désagréments. Cette méthode de travail augmente l'intérêt et l'utilité $\mathrm{du}$ travail et renforce le sentiment d'efficacité, car elle est perçue comme la plus 
pertinente à long terme. La valeur vérité est directement liée au domaine d'activité (projets publics, développement durable), car selon les salariés la vérité ne pourrait pas primer sur la rentabilité dans un autre domaine.

Le second facteur de satisfaction dans l'entreprise privée découle directement de la valeur vérité, de la priorisation du sens sur la rentabilité : il s'agit de l'utilité du travail, mis en avant par quatre opérateurs sur cinq (4/5). Ils ont ainsi le sentiment que leur activité répond à un besoin existant dans la société.

Le troisième facteur de satisfaction (3/5) est la confiance dans les compétences de l'encadrement, qui repose sur la transparence dont fait preuve la gérante aux yeux des opérateurs. La direction les informe du sens qu'elle veut donner à l'activité, et les opérateurs adhèrent pleinement à ces objectifs. Ce sont donc tant les objectifs que le fait qu'ils soient clairement exprimés qui sont sources de satisfaction.

Si la culture d'entreprise est la source principale de satisfaction dans l'entreprise privée, elle s'accompagne d'exigences nouvelles et d'inconvénients qui constituent la source d'insatisfaction la plus citée (3/5). C'est en premier lieu son caractère nouveau et peu répandu qui peut entraîner des difficultés. Le positionnement professionnel entraîné par la culture de vérité reste marginal dans le métier. Il est plus exigeant, car l'opérateur doit sans cesse convaincre ses interlocuteurs de sa pertinence. Alors que la pierre angulaire de l'activité de l'entreprise est d'amener experts et communicants à travailler de manière transversale, cette démarche se freine elle-même, car elle est à la fois trop neuve et trop complexe. La transposition des valeurs de l'entreprise dans l'activité de travail a donc un revers, elle provoque insécurité et stress: «La liberté c'est l'insécurité», dit un opérateur. Cependant, selon les opérateurs, les satisfactions qu'ils retirent de ce positionnement professionnel sont bien plus importantes que les insatisfactions. Mais la culture d'entreprise a aussi des inconvénients au sein du collectif de travail, à travers sa dimension interdisciplinarité. Étant donné la taille de l'entreprise, chaque opérateur est le seul représentant de son métier, ce qui est source d'isolement. Les conséquences négatives du positionnement de l'entreprise sont par ailleurs le seul facteur d'insatisfaction récurrent.

31 Le tableau suivant (tableau 1) synthétise les principaux facteurs de satisfaction et d'insatisfaction dans les deux entreprises.

Tableau 1. Synthèse des satisfactions et insatisfactions des salariés des deux entreprises

\begin{tabular}{|l|l|l|}
\hline & SCOP & Entreprise privée \\
\hline \multirow{5}{*}{ Satisfactions } & $\begin{array}{l}\text {-Cohésion du collectif de } \\
\text { travail (6/6) } \\
\text {-Structure coopérative (5/6) } \\
\text {-Intérêt, utilité et diversité du } \\
\text { travail (3/6) }\end{array}$ & $\begin{array}{l}\text {-Culture d'entreprise : vérité, complémentarité, } \\
\text { liberté (5/5) }\end{array}$ \\
-Utilité du travail (4/5) \\
-Confiance en l'encadrement (3/5) \\
\hline
\end{tabular}




\begin{tabular}{|c|c|c|}
\hline Insatisfactions & $\begin{array}{l}\text {-Déplacements tardifs et } \\
\text { déséquilibre travail/vie privée } \\
(4 / 6) \\
\text {-Cohésion inégale et } \\
\text { envahissante }(2 / 6) \\
\text {-Répartition des tâches }(2 / 6) \\
\text {-Rémunération }(2 / 6)\end{array}$ & $\begin{array}{l}\text {-Exigences et inconvénients du positionnement } \\
\text { de l'entreprise (3/5) : nouveauté, complexité, } \\
\text { isolement }\end{array}$ \\
\hline
\end{tabular}

\title{
3.2 Analyse des scores de satisfaction auto-rapportés
}

\begin{abstract}
Nous demandions également aux participants de situer leur niveau global de satisfaction/ insatisfaction au travail sur une échelle variant de 1 (insatisfaction totale) à 100 (satisfaction totale). Les niveaux de satisfaction au travail relevés au sein de la SCOP varient de 70 à 90 . Ceux relevés au sein de l'entreprise privée varient de 45 à 90 : un salarié a exprimé un score de 45/100 et les scores des autres salariés de l'entreprise varient entre 80 et 90/100.

La satisfaction au travail exprimée est élevée au sein de la coopérative, et c'est également majoritairement le cas au sein de l'entreprise privée, excepté pour un salarié qui se déclare plutôt insatisfait. Lors des entretiens, cette personne avait indiqué des sources d'insatisfaction qu'elle jugeait "conséquentes" et qui n'ont pas été soulevées par d'autres membres de l'entreprise privée, à savoir la faible rémunération et la sousutilisation des compétences personnelles. Ce salarié occupe le poste le moins élevé au sein de la hiérarchie des postes de l'entreprise et juge que les autres ne lui laissent pas l'espace suffisant pour se réaliser pleinement au sein de son activité.
\end{abstract}

\section{Discussion}

\subsection{Les principes d'économie sociale sont sources de satisfaction au travail}

34 L'hypothèse selon laquelle les finalités et modes d'organisation du travail de l'économie sociale seraient sources de satisfaction reposait sur deux éléments principaux. Premièrement, on s'attendait à ce que les principes d'économie sociale - en réduisant la frontière entre conception et exécution et en visant une plus grande maîtrise du processus de travail par les salariés - aient des effets positifs sur la satisfaction tirée de l'activité réalisée : augmentation de l'intérêt du travail, développement des sentiments d'autonomie et de responsabilité, meilleure utilisation des compétences personnelles. Deuxièmement, le fonctionnement collégial était censé renforcer la satisfaction relative au collectif de travail, à l'organisation et à la culture d'entreprise : développement de la cohésion du collectif de travail, de la coopération, et du sens du travail en lien avec l'application de la triple responsabilité de la coopérative (économique, sociale et environnementale). Les informations issues des entretiens confirment le fait que les finalités et modes d'organisation de l'économie sociale sont sources de nombreuses satisfactions professionnelles. La cohésion de l'équipe, les spécificités de la structure coopérative elle-même (responsabilités partagées, gouvernance collégiale, égalité entre salariés, priorisation de l'humain) et l'intérêt et l'utilité de l'activité de travail sont les 
trois principaux facteurs de satisfaction des salariés de la SCOP. Celle-ci est perçue comme source de responsabilités, de coopération, d'autonomie, de considération, d'égalité et de gouvernance collégiale. L'utilité du travail, mise au même niveau que l'intérêt propre pour le travail dans la détermination de la satisfaction ressentie, est présentée comme la conséquence de ce qui fait l'originalité de l'économie sociale: la triple responsabilité de l'entreprise.

Nous parvenons ainsi à des conclusions similaires à celles auxquelles sont récemment parvenus De Cooman, De Gieter, Pepermans et Jegers (2011) à savoir que les salariés des organisations à but non lucratif accordent une valeur prépondérante à l'utilité sociale de leur activité, et montrent un niveau élevé de motivation intrinsèque. En effet, les sources de satisfaction que procurent l'application des principes soutenus par l'économie sociale en SCOP renvoient aux mêmes aspects du travail que ceux établis par la théorie de l'autodétermination comme permettant d'augmenter la satisfaction des trois besoins fondamentaux que sont l'autonomie, la compétence et l'appartenance et donc de susciter de la motivation intrinsèque et de la motivation extrinsèque auto-régulée (Gagné et Forest, 2009) : participation aux décisions, maitrise du processus et des finalités du travail, coopération et partage des connaissances. Or, ces formes de motivation autonome ont été démontrées comme étant liées à de nombreuses conséquences positives (Gagné et Forest, op.cit.; Vallerand, 1997), tant pour les individus au travail que pour leur organisation: «les environnements de travail et les méthodes de management qui supportent l'autonomie (plutôt que le contrôle externe) favorisent la satisfaction des besoins fondamentaux, la motivation intrinsèque et la pleine internalisation des motivations extrinsèques, ce qui à son tour mène à la persistance des comportements, à la performance effective, à la satisfaction au travail, à des attitudes positives au travail, à l'engagement organisationnel et au bien-être psychologique» (Gagné et Deci, 2005, p. 346).

\subsection{Les principes d'économie sociale ne sont pas la propriété exclusive des SCOP}

Si les coopérateurs mettent en avant l'utilité du travail, la culture d'entreprise, la considération, les salariés de l'entreprise privée considérée ici le font tout autant. La culture d'entreprise et l'utilité du travail sont les deux principales sources de satisfaction des salariés de l'entreprise privée. Or ce qui est intéressant, c'est qu'en examinant les valeurs de la culture d'entreprise qui sont sources de satisfaction, on remarque que deux des trois sources de satisfaction principales sont des principes soutenus par l'économie sociale que l'entreprise privée a choisi d'appliquer également. La première de ces valeurs est ce que les salariés appellent la liberté et qui se manifeste par une soumission de la rentabilité à d'autres critères jugés plus importants dans les processus de prise de décision (principe d'économie sociale) et par une application de la liberté à l'interne. La seconde valeur est ce que les salariés appellent la vérité et qui correspond au fait de faire primer le principe de responsabilité sociale (et en ce sens s'apparente plus à l'économie sociale qu'à l'économie de marché) puisqu'il s'agit de mettre au premier plan des objectifs de la communication, la priorisation de l'utile aux citoyens sur le rentable. Deux des principales sources de satisfaction dans l'entreprise privée consistent justement en ce que l'on pensait être les spécificités coopératives : priorisation du sens et de l'utilité du travail sur les aspects de rentabilité, renforcement de l'autonomie et considération de l'autre! 
Ainsi, les quatre principes fondateurs de l'économie sociale énoncés par Defourny (1992) qui devaient fonder la distinction entre économie sociale et économie de marché, entre SCOP et entreprise privée, sont en grande partie adoptés par l'entreprise privée, même si son mode de structuration ne le laisse pas paraître. Si l'on se réfère au modèle de Quinn (1988) sur les styles d'organisation et aux travaux du groupe de recherche FOCUS (Muijen et coll., 1992 ; Lemoine, 1998), l'entreprise privée décrite ici possède les caractéristiques mises en avant par Fraccola et Lemoine (2008) comme étant celles des start-up innovantes. Elle se caractérise par un faible niveau de contrôle. Les relations hiérarchiques sont directes et personnalisées et la priorité est donnée à l'obtention d'un bon climat relationnel. Une forte autonomie est laissée aux salariés et la prise d'initiatives est fortement encouragée. Les salariés s'approprient aisément les buts de l'entreprise. Ces caractéristiques distinguent cette entreprise des entreprises privées classiques et la rapprochent des entreprises relevant de l'économie sociale, et des coopératives en particulier. C'est probablement pour ces raisons que les niveaux de satisfaction globale exprimés ne diffèrent pas beaucoup entre les deux échantillons; nous pouvions nous attendre à ce que les conséquences de l'économie sociale s'expriment uniquement via la structure SCOP, alors qu'elles ont aussi eu tendance à s'exprimer par la culture d'entreprise et par le sens donné à l'activité. L'organisation en SCOP, l'appartenance à l'économie sociale et l'adoption "officielle» de ses principes ne sont donc pas des conditions nécessaires pour que les principes d'économie sociale, qu'on les appelle ainsi ou non, guident les pratiques professionnelles et donc la satisfaction au travail qui en découle.

En résumé, l'examen des entretiens semi-directifs plaide dans un seul sens : les finalités d'économie sociale, le sens qu'elles donnent à l'activité, sont sources de satisfaction au travail. Elles apparaissent parmi les sources principales de satisfaction formulées par les participants des deux types d'entreprise et lorsqu'elles sont sources d'insatisfaction, elles sont systématiquement présentées comme secondaires vis-à-vis de la satisfaction qu'elles procurent. La finalité du travail, le sens qu'on lui donne et qu'on véhicule à travers la culture d'entreprise semblent avoir plus d'influence sur la manière de mener l'activité et sur la façon dont elle est vécue par les personnes que le cadre, la structure formelle dans laquelle le travail s'exécute. Comme le disait un opérateur de la coopérative durant les entretiens : «Ce qui importe, c'est la finalité, ce n'est pas la structure ». C'est parce que la finalité donnée au travail par l'organisation (quelle que soit la structure de cette organisation) correspond à l'orientation que l'individu veut donner à son activité qu'il peut y trouver un sens, source essentielle de motivation et de satisfaction au travail (Morin, 1996). Et comme il apparaît nettement dans cette étude, la notion d'utilité (ressentie) du travail joue un rôle central pour que le travail ait un sens aux yeux de la personne qui l'accomplit (Morin, op. cit.).

\subsection{L'application des principes d'économie sociale n'a pas uniquement des conséquences positives}

Sans remettre en cause ce qui a été dit précédemment, nos résultats contribuent également à montrer que l'application des principes soutenus par l'économie sociale n'a pas uniquement des conséquences positives sur la satisfaction des opérateurs. Si l'application de ces principes est source de nombreuses satisfactions professionnelles dans les deux entreprises, celles-ci ont quasi systématiquement leurs revers : isolement, 
marginalisation, exigences supplémentaires de l'activité. Il est à ce titre remarquable que ce sont les mêmes facteurs qui sont à la fois sources de satisfaction et d'insatisfaction. Ces observations n'abondent pas dans le sens de la théorie bi-factorielle d'Herzberg (1966, 1978) affirmant que les déterminants intrinsèques sont sources de satisfaction tandis que les déterminants extrinsèques sont sources d'insatisfaction. Plus globalement, elles remettent en cause le caractère univoque des déterminants, qui seraient soit sources de satisfaction, soit sources d'insatisfaction, comme le postulent par exemple l'immense majorité des outils de mesure de la satisfaction par questionnaire. Ces résultats révèlent en réalité le caractère polydoxique des attitudes exprimées (Bely, Lemoine et Germain, 1997), ici à l'égard du travail.

\subsection{La structure SCOP aurait-elle une influence propre sur la satisfaction au travail?}

Le fonctionnement coopératif aurait-il des conséquences qui ne résulteraient pas uniquement de l'application des finalités de l'économie sociale, qui ne pourraient être véhiculées par la seule culture d'entreprise et les buts donnés à l'activité ?

La gouvernance collégiale est en effet une source de satisfaction au travail importante au sein de la SCOP examinée ici. Cependant, la gouvernance collégiale est-elle nécessairement gage de satisfaction et de bon fonctionnement? Est-elle applicable et appliquée dans toutes les SCOP ? L'analyse des entretiens réalisés avec les membres de la coopérative apporte quelques pistes de réponse qu'il conviendra d'examiner lors de futures recherches. Les opérateurs interrogés ici, ayant travaillé à la fois en entreprise privée et en SCOP, affirment que l'état de cohésion du collectif de travail est très important pour savoir si la gouvernance collégiale sera davantage source de satisfaction ou d'insatisfaction, de réussite ou d'échec. La cohésion du collectif de travail - entendue ici comme le degré de proximité perçue entre les membres et le niveau de convergence de buts et de valeurs - est perçue par ces salariés comme un prérequis de la collégialité, plutôt que comme une conséquence. Selon eux, la collégialité ne peut fonctionner que si la cohésion est suffisamment forte pour que les salariés aient le sentiment d'être égaux ; Les opérateurs de la SCOP expliquent par exemple que la gestion collective ne s'est réellement affirmée qu'après le départ du créateur. Bien que l'entreprise soit une SCOP depuis sa création, la présence de son créateur dans le collectif avait pour effet d'entraîner les autres collaborateurs à suivre ce "mentor", ce qui restreignait la collégialité effective malgré le statut officiel de coopérative. Lorsque le collectif de travail n'est pas cohésif dans le sens homogène, la collégialité ne semble pas pouvoir s'affirmer, même quand la structure d'entreprise la valorise. Le sentiment d'égalité entre opérateurs, qui repose sur l'impression que les apports de chacun se valent, serait ainsi la pierre angulaire de la bonne adaptation de la collégialité dans un collectif de travail donné. Les individus vont utiliser des éléments de comparaison tels que l'âge ou le niveau d'expérience pour juger s'ils apportent plus ou moins qu'un autre au collectif. S'ils en arrivent à la conclusion qu'ils sont relativement égaux quant à leurs apports, ils ressentiront certainement le besoin que l'organisation reflète cette égalité en leur donnant le même statut. À l'inverse, si les salariés ne se sentent pas égaux, s'ils pensent que leurs contributions sont inégales, ils ne souhaiteront probablement pas qu'une organisation collégiale vienne gommer ces différences qui existent " réellement " (au moins dans les processus de jugement des individus). Le coopératisme ne peut donc 
décréter l'égalité entre opérateurs nécessaire à son fonctionnement; il faut que l'égalité trouve sa légitimité dans l'homogénéité du collectif de travail pour que la collégialité puisse venir formaliser et s'appuyer sur l'égalité qui la précède. À l'inverse, lorsque les salariés se considèrent égaux et que ce sentiment n'est pas renforcé par une structure collégiale mais contredit par un système pyramidal, des insatisfactions sont susceptibles de naître. La structure collégiale ne serait donc pas en soi source de satisfaction, pas plus que la structure pyramidale. C'est de l'adaptation de la structure choisie aux caractéristiques du collectif de travail que dépendrait le succès, en matière de satisfaction, d'un mode de structuration donné. On peut par ailleurs penser que les deux dimensions s'entretiennent mutuellement: si la cohésion est un prérequis à la collégialité, le système coopératif renforce également la cohésion, notamment à travers le recrutement: on recherche des collaborateurs qui, outre leurs compétences professionnelles, présentent une capacité à soutenir les valeurs de l'entreprise.

\subsection{Conclusion générale}

41 En conclusion, cette étude a contribué à montrer que les principes soutenus par les organisations relevant de l'économie sociale, et par les SCOP en particulier, ont un effet positif sur la satisfaction au travail. Celui-ci réside dans l'adhésion des salariés à ces principes, qu'on les nomme "d'économie sociale » ou non : utilité plutôt que rentabilité, autonomie en interne comme à l'externe, processus de décision démocratique, réduction de la séparation entre conception et exécution. Par ailleurs, l'impact de ces principes sur les caractéristiques du travail, et par conséquent sur les attitudes des individus à l'égard de ce travail, peut être véhiculé par les buts donnés au travail et la culture d'entreprise et n'est pas strictement dépendant du type de structure de travail mise en place (SCOP). En d'autres termes, les résultats de la présente recherche tendent à démontrer que si l'adoption et la mise en application, par une majorité des entreprises, des valeurs défendues par l'économie sociale serait sans nul doute vecteur de satisfaction au travail chez les salariés - notamment en ce qui a trait au développement de l'intérêt et du sens $\mathrm{du}$ travail - l'adoption d'une gouvernance collégiale doit, elle, dépendre des caractéristiques du collectif de travail.

Nous tenons à remercier les salariés des deux entreprises participantes pour avoir accepté de prendre part à cette étude. Nous remercions également vivement Eleanor Moody pour son aide précieuse dans la relecture de la version anglophone de cet article.

\section{BIBLIOGRAPHIE}

Alternatives économiques. (2006). L'économie sociale de A à Z. Guide pratique d'Alternatives économiques, 22 (janvier).

Argyle, M. (2001). The Psychology of Happiness ( $\left.2^{\text {nd }} e d.\right)$. London: Routledge. 
Belly, B., Lemoine, C. et Germain, R. (1997). La polydoxie concernant l'autorité : multiplicité d'opinions en fonction de la méthode de leur recueil. Psychologie du travail et des organisations, 3 , 1-2, 20-31.

Bretz, R. D. et Judge, T. A. (1994). Person-organization fit and the theory of work adjustment: Implications for satisfaction, tenure, and career success. Journal of Vocational Behavior, 44(1), 32-54. Centre d'analyse stratégique (2009). La santé mentale, l'affaire de tous. Pour une approche cohérente de la qualité de vie. Rapport du Centre d'analyse stratégique, 115 (nov.) www.strategie.gouv.ff

CNCRES (2009). Atlas de l'économie sociale et solidaire 2009. http://www.rtes.fr/IMG/pdf/ AtlasESSFranceRegionsCNCRES.pdf

Dawis, R. V. (1992). Person-environment fit and job satisfaction. In C. J. Cranny, P. C. Smith et E. F. Stone (Eds.), Job satisfaction: How people feel about their jobs and how it affects their performance. New York: Lexington Books.

De Cooman, R., De Gieter, S., Pepermans, R. et Jegers, M. (2011). A cross-sector comparison of motivation related concepts in for profit and not for profit service organizations. Nonprofit and Voluntary Sector Quarterly.40(2), 296-317.

Defourny, J. (1992). L'émergence du secteur d'économie sociale en Wallonie. Coopératives et développement, 23(1), 151-175.

Durand-Delvigne, A., Verquerre, R. et Masclet, G. (2000). L'aménagement et la réduction du temps de travail : quels effets sur la qualité de vie ? Psychologie du travail et des organisations, 6(1-2), 107-119.

Fraccola, R. et Lemoine, C. (2008). Start-up innovantes : conséquences de l'évolution organisationnelle sur la culture des salariés et le climat de travail. In C. Lemoine, A. Balikdjian, N. Kridis et $\mathrm{P}$. Salengro (Eds.), Organisation, engagement et équipes de travail. Actes du XIV ${ }^{e}$ Congrès de l'AIPTLF, tome 5 (p.88-99). Lille : Éditions de l'AIPTLF (CD-ROM).

Gagné, M. et Deci, E. L. (2005). Self-determination theory and work motivation. Journal of Organizational Behavior, 26, 331-362.

Gagné, M. et Forest, J. (2009). La motivation au travail selon la théorie de l'autodétermination, vol. 3. In P. Roussel, J. Rojot et C. Vandenberghe (Eds.), Comportement organisationnel (p. 215-234). Bruxelles : Éditions De Boeck.

Herzberg, F. (1966). Le travail et la nature de l'homme. 4 e édition en 1978. Paris : Entreprise Moderne d'Édition.

Iaffaldano, M. T. et Muchinsky, P. M. (1985). Job satisfaction and job performance: A metaanalysis. Psychological Bulletin, 97(2), 251-273.

Johnson, G. et Johnson, W. (2002). Perceived over-qualification and dimensions of job satisfaction: A longitudinal analysis. Journal of Psychology, 134, 537-555.

Kristof-Brown, A. L., Zimmerman, R. D. et Johnson, E. C. (2005). Consequences of individuals' fit at work: A meta-analysis of person-job, person-organization, person-group, and person-supervisor fit. Personnel Psychology, 58, 281-342.

Lemoine, C. (1998). Style d'organisation et mode d'évaluation. In M. Rousson et L. ThygesenFischer (Eds.), Psychologie du travail et transformations de la société (p. 99-107). Neuchâtel : Presses Académiques de Neuchâtel. 
Lemoine, C. (2007). De la question à l'autoquestionnement : questionner, source d'emprise et d'appropriation multiple. Psychologie du travail et des organisations, 13, $n^{\circ} 4$, p. 5-24.

Morin, E. M. (1996). Psychologies au travail. Montréal : Gaétan Morin Éd.

Muijen, J. J. Van, Koopman, P., Dondeyne, P., De Cock, G. et De Witte, K. (1992). Organizational culture, the development of an international instrument for comparing countries. Proceedings $2^{\text {nd }}$ congress of psychology. Budapest.

Quinn, R. (1988). Beyond rational management. San Francisco : Jossey-Bass.

Réseau SCOP entreprises (2010). Chiffres des SCOP.

http://www.les-scop.coop/sites/fr (date de consultation : 6/08/10).

Ritter, A. J. et Anker, R. (2002). Bons emplois, mauvais emplois : les jugements des travailleurs dans cinq pays. Revue internationale du travail, 141(4), 331-358.

Souza-Pouza, Al. et Souza-Pouza, An. (2000). Well-being at work: A cross-national analysis of the levels and determinants of job satisfaction. Journal of Socio-Economics, 29, 517-538.

Spector, P. E. (1997). Job satisfaction: Application, assessment causes and consequences. London: Sage.

Tett, R. P. et Meyer, J. P. (1993). Job satisfaction, organizational commitment, turnover intention, and turnover: Path analyses based on meta-analytic findings. Personnel Psychology, 46(2), 259-293.

Vallerand, R. J. (1997). Toward a hierarchical model of intrinsic and extrinsic motivation. In M. P. Zanna (Ed.), Advances in experimental social psychology (vol. 29, p. 271-360). San Diego, CA: Academic Press.

Vandenberghe, C., Stordeur, S. et D'Hoore, W. (2009). Une analyse des effets de la latitude de décision, de l'épuisement émotionnel et de la satisfaction au travail sur l'absentéisme au sein des unités de soins infirmiers. Le travail humain, 72(3), 209-228.

Van Knippenberg, D. et Van Schie, C. M. (2000). Foci and correlates of organizational identification. Journal of Occupational and Organizational Psychology, 73(2), 137-147.

Weiss, H. M. (2002). Deconstructing job satisfaction: Separating evaluations, beliefs, and affective experiences. Human Resource Management Review, 12, 1-22.

\section{RÉSUMÉS}

Nous étudions les effets de l'économie sociale sur la satisfaction au travail. Selon l'hypothèse, l'adoption des finalités et des modes d'organisation du travail de l'économie sociale est source de satisfaction au travail : réduction de la séparation entre conception et exécution, augmentation de l'intérêt et de l'utilité perçue du travail, meilleure cohésion et coordination entre les travailleurs. Les résultats de l'analyse de contenu des entretiens semi-directifs menés auprès des salariés de deux entreprises exerçant dans le secteur du conseil en développement durable en France, l'une société coopérative (SCOP) et l'autre entreprise privée, confirment cette hypothèse. Le fait que les principes d'économie sociale ont des conséquences positives sur la satisfaction au travail dans les deux entreprises montre par ailleurs que l'influence positive de l'économie sociale sur le bien-être au travail dépend moins des modes de structuration formels que du sens donné au travail.

This study investigates the impact of social economics on job satisfaction. According to the hypothesis, the adoption of social economics' goals and modes of work organization is a source of job satisfaction: reduction in the separation between conception and execution, increase in 
work's interest and perceived usefulness, and better cohesion and coordination among workers. Semi-structured interviews were conducted with the workers in two consulting firms in sustainable development in France, one a cooperative and the other a private company. The results of the content analysis corroborate the hypothesis. The fact that social economy's principles have positive impacts on job satisfaction in both companies also shows that social economy's positive influence on well-being at work depends less on formal work organization than on the meaning given to work.

Este artículo estudia los efectos de la economía social sobre la satisfacción en el trabajo. Según la hipótesis, la adopción de los objetivos y los modos de organización laboral de la economía social es una fuente de satisfacción en el trabajo : reducción de la brecha entre la concepción y la ejecución, aumento del interés y de la percepción de utilidad del trabajo, mejora de la cohesión y la coordinación entre los trabajadores. Se realizaron entrevistas semi-dirigidas a asalariados de dos empresas consultoras en desarrollo sostenible en Francia, la primera una sociedad cooperativa (SCOP) y la otra una empresa privada, los resultados del análisis del contenido de estas entrevistas confirman la hipótesis.

El hecho de que los principios de economía social tuvieron un impacto positivo sobre la satisfacción en el trabajo dentro de las dos empresas muestra, además, que la influencia positiva de la economía social sobre el bienestar en el trabajo depende menos de la forma organización en trabajo que del sentido dado al mismo.

\section{INDEX}

Mots-clés : satisfaction au travail, économie sociale, coopérative, sens du travail, organisation du travail

Keywords : job satisfaction, social economy, cooperative, work purposes, work organization Palabras claves : satisfacción en el trabajo, economía social, cooperativa, sentido del trabajo, organización laboral

\section{AUTEURS}

\section{DAVY CASTEL}

davy.castel@univ-lille3.fr, Univ Lille Nord de France, F-59000 Lille, France, UDL3, PSITEC, F-59653 Villeneuve d'Ascq, France

\section{CLAUDE LEMOINE}

claude.lemoine@univ-lille3.fr, Univ Picardie Jules Verne, LPA, F-80025 Amiens, France

\section{ANNICK DURAND-DELVIGNE}

annick.durand-delvigne@univ-lille3.fr, PSITEC - E.A. 4072, Univ Lille Nord de France, F-59000

Lille, France, UDL3, PSITEC, F-59653 Villeneuve d'Ascq, France 